\title{
Lymphopenia and lung complications in patients with coronavirus disease-2019 (COVID-19): A retrospective study based on clinical data
}

\author{
Ehsan Zaboli ${ }^{1}$, Hadi Majidi ${ }^{1}$, Reza Alizadeh-Navai ${ }^{2}$, Akbar Hedayatizadeh-Omran ${ }^{2}$, \\ Hossein Asgarian-Omran ${ }^{1}$, Laleh Vahedi Larijani ${ }^{1}$, Vahid Khodaverdi ${ }^{1}$, and Omolbanin \\ Amjadi $^{3}$ \\ ${ }^{1}$ Mazandaran University of Medical Sciences Faculty of Health \\ ${ }^{2}$ Mazandaran University of Medical Sciences Faculty of Medicine \\ ${ }^{3}$ Affiliation not available
}

January 6, 2021

\begin{abstract}
Background: A rapid outbreak of novel coronavirus, COVID-19, made it a global pandemic. This study focused on the possible association between lymphopenia and Computed tomography (CT) scan features and COVID-19 patient mortality. Method: The clinical data of 596 COVID-19 patients were collected from February 2020 to September 2020. The patients' serological survey and CT scan features were retrospectively explored. Results: The median age of the patients was $56.7 \pm 16.4$ years old. Lung involvement was more than $50 \%$ in 214 COVID-19 patients (35.9\%). The average blood lymphocyte percentage was 20.35 \pm 10.16 . The levels of C-reactive protein (CRP), erythrocyte sedimentation rate (ESR), and platelet-to-lymphocyte ratio (PLR) may not indicate the severity and prognosis of COVID-19. Patients with severe lung involvement and lymphopenia were found to be significantly associated with increased odds of death (odds ratio [OR], 9.24; 95\% confidence interval [95 CI\%], 4.32- 19.78). These results indicated that lymphopenia $<20 \%$ along with pulmonary involvement $>50 \%$ impose a multiplicative effect on the risk of mortality. The in-hospital mortality rate of this group was significantly higher than other COVID-19 hospitalized cases. Furthermore, they meaningfully experienced a prolonged stay in the hospital $(\mathrm{P}=0.00)$. Conclusion: The Lymphocyte count less than $20 \%$ and chest CT scan findings with more than $50 \%$ involvement might be related to the patient's mortality. It could act as laboratory and clinical indicators of disease severity and mortality.
\end{abstract}

\section{Introduction}

In December 2019, pneumonia for unknown reasons emerged in Wuhan city, Hubei of China. Chinese scientists extracted novel Coronavirus from patients' specimens and named it as severe acute respiratory syndrome coronavirus 2 on 7 January 2020 that was formerly called N-cov-2019 ${ }^{1}$. The World Health Organization (WHO) finally announced the new coronavirus as COVID-19 in February $2020^{1}$. Coronaviruses are a large family of viruses that are well-known to cause a wide variety of clinical manifestations from the mild common cold to severe forms of respiratory diseases such as Middle East Respiratory Syndrome (MERS) and severe acute respiratory syndrome (SARS) ${ }^{2,3}$. The clinical manifestations of COVID-19 vary from asymptomatic infection, mild forms of upper respiratory tract illness, viral pneumonia, acute respiratory failure, and death ${ }^{4,5}$. The most common clinical features of COVID-19 including fever, dry cough, fatigue, diarrhea, severe muscle pain, and pneumonia that lead to Acute Respiratory Distress Syndrome (ARDS), metabolic acidosis, septic shock, thrombosis, and heart failure, renal failure, and liver disease ${ }^{6,7}$. Although the mild form of COVID-19 can be treated by appropriate medical interventions, the most severe cases especially the elderly who experience underlying medical conditions develop severe illness and increase the mortality rate ${ }^{8}$. 
Older adults with pre-existing medical conditions such as diabetes, asthma, and cardiovascular disease may be more vulnerable to COVID-19 ${ }^{9}$. The recognition and discrimination of severe COVID-19, mild infection, and asymptomatic form are clinically vital ${ }^{10}$. The most important laboratory findings in COVID-19 patients are low concentration of albumin (75.8\%), high serum C-reactive protein (CRP) (58.3\%), ESR (41.8\%), LDH $(57 \%)$, and lymphopenia (43\%) ${ }^{11}$. Lymphopenia was detected in severe COVID-19 patients (85\%) and suggested as a severity predictor; the average count of lymphocytes of Intensive Care Unit (ICU) - hospitalized patients was $800^{12}$. Lymphopenia could have occurred in COVID-19 patients via four mechanisms: a) viral attachment to the cell surface receptor ACE2, infect lymphocytes that lead to lymphocyte death ${ }^{13}$; b) the possible role of coronavirus in the destruction of lymphoid organs ${ }^{14}$; c) induction of lymphocyte apoptosis by the production of TNF- $\alpha$ and IL- $6^{15}$ and d) inhibition of lymphocyte production during metabolic acidosis ${ }^{16}$. Although the pathogenesis of COVID-19 remains unclear, lymphopenia was observed in most of the patients ${ }^{17}$. Aging and chronic illness lead to endothelial dysfunction that dismounts cell-cell adhesions, promotes endothelial cell death, extravasation that resulted in lymphopenia ${ }^{18}$. The infection of COVID-19 is diagnosed and confirmed by Real-Time PCR (RT-PCR) and gene sequencing of the blood and lung samples. In the early phase of the disease, positive nasopharyngeal RT-PCR results ranged between $30-60 \%{ }^{19}$. In emergency cases, the low sensitivity of RT-PCR misses diagnoses of COVID-19 patients failed receiving appropriate medications and upended outside spreading of COVID-19. In contrast with RT-PCR, chest computed tomography (CT) has sufficient sensitivity for atypical radiographic manifestations of COVID-19 cases who are asymptomatic and/or negative on the initial RT-PCR test ${ }^{20,21}$. According to the Chinese reports, chest CT is adequately sensitive (97\%) in early detection of COVID- 19 patients compared with RT-PCR ${ }^{22}$. Bilateral lung involvement was observed in $98 \%$ of patients and lobular and subsegmental areas of consolidation were considered as the most typical findings of $\mathrm{CT}^{4}$. Besides, several COVID-19 cases demonstrated ground-glass opacities (GGO) and pulmonary consolidation with round morphology ${ }^{23}$. The main CT findings of COVID-19 pneumonia are the shape of GGO, crazy paving pattern, and Consolidation ${ }^{24}$.

Iran is one of the worst affected countries by the coronavirus and Mazandaran province is considered one of the most impacted areas ${ }^{25}$. The paucity of evidence on the relationship between lymphopenia, chest CT examination, and mortality rate in COVID-19 patients made us ascertain this possibility. Since Imam Khomeini hospital in Mazandaran was considered as the main referral center for the management of COVID19 cases, we aim to retrospect and evaluate the lymphopenia in COVID-19 patients and its association with lung involvement.

\section{Methods}

\section{Study Design and Inclusion Patients}

This study was approved by the research ethics committee Mazandaran University of Medical Sciences, Sari, Iran (IR.MAZUMS.REC.1399.7418). All patients who were admitted to Emam Khomeini hospital, Sari, Mazandaran from February 26, 2020, to September 21, 2020, were included. The written informed consent was given up from all participants with COVID-19 infection. Simple random sampling was used for including patients. The diagnosis, hospitalization, and treatment of patients was performed according to Iran's Ministry of Health and Medical Education guidelines. Iranian guidelines mentioned the laboratory and radiological examinations as a first line of COVID-19 confirmation along with clinical symptoms such as fever, cough, and shortness of breath. The cases without chest $\mathrm{CT}$ and laboratory test confirmation were excluded. Assuming 95\% confidence level and $\mathrm{p}=0.7$ (lymphopenia 70\%), $\mathrm{d}=0.07$, and Cochran's formula the sample size was calculated as 596. Clinical outcomes including CBC, CRP, and ESR were evaluated within a 7-days monitoring period. All medical data including epidemiological, demographic, laboratory data, and patient's discharge and/or death were extracted via the hospital information system (HIS).

\section{CT scans}

All CT examinations were performed using a multi-detector computed tomography scanner (CT-Scan) Somatom Emotion eco (16-slice configuration, Siemens, Erlangen, Germany). The supine CT scan was performed and no contrast medium had been administrated. The CT images were observed with both lungs and 
in parenchymal and mediastinal window settings. Two radiologists appraised the lung scans by the picture archiving and communication system (PACS, INFINITT Healthcare co., Ltd., Seoul, Korea). The features of CT imaging were typically focused on unilateral and bilateral Ground Glass Opacity (GGO), consolidation, and pleural effusion. The pulmonary involvement of COVID-19 patients was categorized into two groups: less than 50\% (Figure 1-A) and more than $50 \%$ involvement (Figure 1-B). Clinical data were screened on the date of symptom onset and $7^{\text {th }}$ day and abstracted from the medical records of Imam Khomeini hospital, Sari, Iran. All data containing age, sex, underlying medical conditions, CT scan results, lymphocyte count, CRP, ESR, Platelet, length of stay (LOS), and mortality was recorded according to the checklist. Data analysis was performed using SPSS, and Mann-Whitney and Kruskal-Wallis tests. The logistic regression model interprets the odds ratio and $\mathrm{P}<0.05$ was considered as statistically significant.

\section{Results}

A total of 596 patients were included. The median age was $56.7 \pm 16.4$ years (range 18-94 years), and 300 patients $(50.3 \%)$ were male and 296 patients $(49.7 \%)$ were female. Based on the CT findings categorization, 214 patients $(35.9 \%)$ presented $>50 \%$ lung involvement; 84 deaths occurred $(14.09 \%)$ and $85.9 \%$ of patients were discharged. The mean blood lymphocyte percentage was $20.35 \pm 10.16$ (Table 1). The mean lymphocyte percentage of COVID-19 patients with $>50 \%$ lung involvement was significantly lower than that of subjects $<$ $50 \%$ involvement (median of 17.36 vs. 22.03) ( $\mathrm{p}=0.00$ ) (Figure 2). All patients were stratified into four groups according to the radiographic and hematological findings: 74 patients $(12.4 \%)$ with $>50 \%$ lung involvement and without lymphopenia; 210 patients $(35.2 \%)<50 \%$ lung involvement and without lymphopenia; 140 patients $(23.5 \%)>50 \%$ lung involvement and with lymphopenia, and 172 patients $(28.9 \%)<50 \%$ lung involvement and with lymphopenia. The mortality was intensely found to be correlated with lung involvement and lymphopenia in COVID-19 patients (Table 2). The mortality rate of patients who presented $>50 \%$ lung involvement was higher than patients without lymphopenia and lung involvement (OR 3.4, [95\% CI 1.3-8.9]). Those with lymphopenia had a $10.5 \%$ mortality rate (OR 3.62, [95\% CI 1.6-8.0]). COVID-19 patients with lymphopenia and lung involvement showed the highest mortality rate of 36.6\% (OR 9.2, [95\% CI 4.32-19.78]). Length of stay (LOS) of patients with lymphopenia and developed severe lung involvement was correlated strongly with normal lymphocyte count and without pulmonary involvement $(\mathrm{p}=0.000)$ (Table 3$)$. The LOS of patients with lung involvement and lymphopenia was significantly higher than those with lymphopenia $(\mathrm{p}=0.014)$. The inflammatory marker of platelet-to-lymphocyte ratio (PLR) was also analyzed; the median PLR of all patients was 179.58 (IQR [127.84- 245.68]. Of all COVID-19 patients with lung involvement, the mean of PLR in death cases was not significantly associated with the discharged ones $(\mathrm{p}=0.16)$. A significant correlation was not found between PLR and length of stay (LOS) ( $\mathrm{p}=0.062$; Spearman correlation coefficient: $0.077)$.

\section{Discussion}

This research aims to inquire about the association between lymphopenia and lung involvement in the outcome of hospitalized patients with COVID-19. Here, the lymphocyte percentage was calculated as 20.35 \pm 10.16 , it was significantly associated with more severe lung involvement $(\mathrm{P}=0.00)$. The lymphopenia was observed in 312 patients (52.3\%) and 284 patients lied within the normal range. Of the 596 hospitalized patients, 84 (14\%) died and 515 cases were discharged. We observed that CRP and ESR may not a predictable inflammation marker in patients with COVID-19. Of the 596 patients, 260 (43.6\%) showed bilateral groundglass and consolidative opacities, $253(42.4 \%)$ had bilateral ground-glass opacities, and $50(8.4 \%)$ had bilateral consolidation on chest CT. While the pleural effusion, consolidation, GGO was seen in COVID-19 cases, 24 patients $(4.1 \%)$ had exclusively unilateral lung involvement. The bilateral ground-glass opacities and consolidation were the major pulmonary CT findings of COVID-19 patients.

In the same conclusion as ours, other studies reported lymphopenia as a reliable indicator for COVID-19 severity. Tan et al were shown that there is a reverse association between lymphocyte count and COVID19 severity and its prognosis ${ }^{14}$. The lymphocyte count $<20 \%$ was reported in severe clinical illness; lymphopenia at the level $<5 \%$ was found in patients who died ${ }^{14}$. In another study, lymphopenia at the level of $40 \%$ was demonstrated within 191 patients ${ }^{26}$. It was also presented that $48 \%$ of included patients 
experienced underlying non-communicable diseases including hypertension, diabetes, and coronary heart disease ${ }^{26}$. Several symptoms of coronavirus infection were also described in Iranian children and were defined as a less serious disease with a good prognosis ${ }^{27}$. Zhenget al compared the laboratory features of COVID-19 pneumonia to non- COVID-19 pneumonia. They stated that COVID-19 pneumonia- derived lymphopenia negatively affect the biochemical indicators of organ dysfunction ${ }^{28}$. A study done on 221 hospitalized COVID-19 patients confirmed leukopenia (33\%), lymphopenia (73.8\%), and more susceptibility to fungal and bacterial infections ${ }^{29}$. Chinese research explained that bilateral pneumonia, shorter duration of onset to admission, lymphopenia, and disease severity are regarding as risk factors of prolonged hospitalization of COVID-19 ${ }^{30}$. Lymphopenia may occur along with an increased level of cytokine, disease severity, mortality, and impaired immune response ${ }^{31}$.

Tan et al showed the inverse relationship between lymphocyte count and disease severity and its prognosis in 92 COVID-19 patients ${ }^{32}$. It was disclosed that LYM\% lower than $20 \%$ was seen in severe cases and it fell under $5 \%$ in dead patients ${ }^{32}$. Comparison of laboratory variations of 88 patients with COVID-19 pneumonia and 22 non-COVID-19 pneumonia cases revealed that lymphocyte depletion, not neutrophil, and monocyte, inversely affect biochemistry disorder and organ failure ${ }^{33}$. Lymphopenia and augmentation of cytokine are accompanied by increased disease severity, death, and disrupted immune response ${ }^{31}$. Despite that some studies found higher levels of ESR and CRP as inflammatory indicators in severe patients of COVID-19 $9^{34-36}$, our results did not show increased ESR and CRP levels in severe cases. It was also shown by Peng $e t a l^{37}$.

The chest CT scan plays a crucial role in the early diagnosis and evaluation of patients with COVID-19 pneumonia likewise, lymphopenia and thrombocytopenia were the verified markers for disease detection ${ }^{38}$. The sensitivity of a chest CT imaging was examined among 1014 patients who had negative RT-PCR ${ }^{19}$. Their results showed high sensitivity of lung CT ( $75 \%$ of the included population were positive for COVID19) and suggested it as a reliable detection method in epidemic regions ${ }^{19}$. Subgroup analysis revealed that COVID-19 patients with severe pulmonary lung involvement and lymphopenia had 9.2-fold increased odds of in-hospital mortality. The mortality rate was also calculated in patients with severe lung involvement and patients with lymphopenia, these groups had a mortality rate of 3.4 and 3.6 times greater than those without lung involvement and lymphopenia (Table 3). In other words, the mortality in the case of lymphopenia along with severe lung involvement was deeply different compared with patients with neither lymphopenia nor lung involvement (OR 9.2, [95\% CI 4.3- 19.7]) (Table 3). We found that the incremental effect of lymphocyte count and lung involvement tracks the multiplicative model, no additive model.

Assessment of RT-PCR positive COVID-19 cases showed leukopenia, lymphopenia, and high CRP concentration as hematological and biochemical parameters. The most involved segments were lung segments 6 and 8 with peripheral pulmonary localized lesions ${ }^{39}$. The sensitivity of the CT scan was inspected in mild COVID-19 reach to a high level after 10 days of infection ${ }^{40}$. It was examined that the most frequent CT Manifestations and Clinical Features were GGO $(86 \%)$ and consolidation $(62 \%)^{41}$. Similarly, we found that bilateral distribution of GGO and consolidation are the main pulmonary lesions of COVID-19 patients.

The inflammatory marker of platelet to lymphocyte ratio (PLR) was suggested as a predictive indicator for disease severity and mortality in infectious disease and cancer ${ }^{42,43}$. Rong et al noted the value of PLR for predicting the clinical outcome of COVID-19 and patients' observing. They found out a high PLR is associated with more severity and longer hospitalization ${ }^{44}$. Conversely, our study showed no significance and it was not correlated to mortality $(\mathrm{p}=0.16)$. The PLR was not correlated with the LOS $(\mathrm{p}=0.06 ; \mathrm{r}=0.07)$. Notably, we found that LOS in patients with lymphopenia and severe lung involvement was remarkably higher than others $(\mathrm{p}<0.05)$. Likewise, a Chinese study represented that bilateral pneumonia in a lung CT scan, a short period from symptoms onset to hospitalization, lymphopenia, and disease severity were the main factors in prolonged LOS (ProLOS) ${ }^{45}$.

\section{Limitations}

Limitations of this study include the lack of accessibility to the molecular kit at the beginning of the pandemic. Therefore molecular confirmation of COVID-19 was impossible for all included patients. 


\section{Conclusion}

In summary, while SARS-CoV-2 Real-Time RT-PCR is considered as a gold standard method ${ }^{46}$, it is accompanied by false-negative results and less than $100 \%$ sensitivity ${ }^{47}$. Even though the proper period for sampling is the first week after symptom onset, many cases are asymptomatic carriers with pulmonary involvement. COVID-19 pneumonia displays the lung involvements and lesions even in asymptomatic patients within 1-3 weeks. Besides, blood parameters are easily accessible and commonly used everywhere. Relating the valuation of $\mathrm{CT}$ scan features with clinical and laboratory outcomes possibly assist the timely diagnosis of COVID-19 pneumonia. It was clarified in our study that lymphopenia $<20 \%$ and lung CT involvement $>50 \%$ were intensely related todisease severity, mortality, and prolonged hospitalization.

\section{Declarations}

Funding: The current research was supported by Mazandaran University of Medical Sciences (grant no. MAZUMS. 7418).

Conflicts of interest/Competing interests : The authors declare that they have no conflict of interest.

Ethics approval: The study protocol was approved by the Ethics Committee of Mazandaran University of Medical Sciences (IR.MAZUMS..REC.1399.7418).

\section{Consent to participate:}

Consent for publication:

\section{Availability of data and material:}

\section{References}

1. Phelan AL, Katz R, Gostin LO. The Novel Coronavirus Originating in Wuhan, China: Challenges for Global Health Governance. Jama.2020.

2. de Groot RJ, Baker SC, Baric RS, et al. Middle East respiratory syndrome coronavirus (MERS-CoV): announcement of the Coronavirus Study Group. Journal of virology. 2013;87(14):7790-7792.

3. Cui J, Li F, Shi Z-L. Origin and evolution of pathogenic coronaviruses. Nature reviews Microbiology. 2019;17(3):181-192.

4. Huang C, Wang Y, Li X, et al. Clinical features of patients infected with 2019 novel coronavirus in Wuhan, China. Lancet.2020;395(10223):497-506.

5. Bai Y, Yao L, Wei T, et al. Presumed Asymptomatic Carrier Transmission of COVID-19. Jama. 2020;323(14):1406-1407.

6. Chen N, Zhou M, Dong X, et al. Epidemiological and clinical characteristics of 99 cases of 2019 novel coronavirus pneumonia in Wuhan, China: a descriptive study. The Lancet.2020;395(10223):507-513.

7. Wang D, Hu B, Hu C, et al. Clinical Characteristics of 138 Hospitalized Patients With 2019 Novel Coronavirus-Infected Pneumonia in Wuhan, China. JAMA. 2020;323(11):1061-1069.

8. Wang P, Lu J, Jin Y, Zhu M, Wang L, Chen S. Epidemiological characteristics of 1212 COVID-19 patients in Henan, China.medRxiv. 2020:2020.2002.2021.20026112.

9. Jin Y-H, Cai L, Cheng Z-S, et al. A rapid advice guideline for the diagnosis and treatment of 2019 novel coronavirus (2019-nCoV) infected pneumonia (standard version). Military Medical Research.2020;7(1):4.

10. Tabata S, Imai K, Kawano S, et al. The clinical characteristics of COVID-19: a retrospective analysis of 104 patients from the outbreak on board the Diamond Princess cruise ship in Japan. medRxiv.2020:2020.2003.2018.20038125. 
11. Rodriguez-Morales AJ, Cardona-Ospina JA, Gutierrez-Ocampo E, et al. Clinical, laboratory and imaging features of COVID-19: A systematic review and meta-analysis. Travel Medicine and Infectious Disease.2020;34:101623.

12. Yang $\mathrm{X}, \mathrm{Yu} \mathrm{Y}, \mathrm{Xu}$ J, et al. Clinical course and outcomes of critically ill patients with SARS-CoV2 pneumonia in Wuhan, China: a single-centered, retrospective, observational study. Lancet Respir Med. 2020;8(5):475-481.

13. Xu H, Zhong L, Deng J, et al. High expression of ACE2 receptor of 2019-nCoV on the epithelial cells of oral mucosa. International Journal of Oral Science. 2020;12(1):8.

14. Tan L, Wang Q, Zhang D, et al. Lymphopenia predicts disease severity of COVID-19: a descriptive and predictive study. Signal Transduct Target Ther. 2020;5:33.

15. Liao YC, Liang WG, Chen FW, Hsu JH, Yang JJ, Chang MS. IL-19 induces production of IL-6 and TNF-alpha and results in cell apoptosis through TNF-alpha. J Immunol. 2002;169(8):4288-4297.

16. Fischer K, Hoffmann P, Voelkl S, et al. Inhibitory effect of tumor cell-derived lactic acid on human $\mathrm{T}$ cells. Blood.2007;109(9):3812-3819.

17. Xu Z, Shi L, Wang Y, et al. Pathological findings of COVID-19 associated with acute respiratory distress syndrome. Lancet Respir Med. 2020;8(4):420-422.

18. Bermejo-Martin JF, Martin-Fernandez M, Lopez-Mestanza C, Duque P, Almansa R. Shared Features of Endothelial Dysfunction between Sepsis and Its Preceding Risk Factors (Aging and Chronic Disease). $J$ Clin Med. 2018;7(11).

19. Ai T, Yang Z, Hou H, et al. Correlation of Chest CT and RT-PCR Testing in Coronavirus Disease 2019 (COVID-19) in China: A Report of 1014 Cases. Radiology. 2020:200642.

20. Xie X, Zhong Z, Zhao W, Zheng C, Wang F, Liu J. Chest CT for Typical 2019-nCoV Pneumonia: Relationship to Negative RT-PCR Testing.Radiology. 2020:200343.

21. Huang P, Liu T, Huang L, et al. Use of Chest CT in Combination with Negative RT-PCR Assay for the 2019 Novel Coronavirus but High Clinical Suspicion. Radiology. 2020;295(1):22-23.

22. Lee EYP, Ng M-Y, Khong P-L. COVID-19 pneumonia: what has CT taught us? The Lancet Infectious Diseases. 2020;20(4):384-385.

23. Chung M, Bernheim A, Mei X, et al. CT Imaging Features of 2019 Novel Coronavirus (2019-nCoV). Radiology. 2020;295(1):202-207.

24. Koo HJ, Lim S, Choe J, Choi S-H, Sung H, Do K-H. Radiographic and CT Features of Viral Pneumonia. RadioGraphics. 2018;38(3):719-739.

25. Arab-Mazar Z, Sah R, Rabaan AA, Dhama K, Rodriguez-Morales AJ. Mapping the incidence of the COVID-19 hotspot in Iran - Implications for Travellers. Travel medicine and infectious disease.2020;34:101630-101630.

26. Zhou F, Yu T, Du R, et al. Clinical course and risk factors for mortality of adult inpatients with COVID-19 in Wuhan, China: a retrospective cohort study. Lancet. 2020;395(10229):1054-1062.

27. Rahimzadeh G, Ekrami Noghabi M, Kadkhodaei Elyaderani F, et al. COVID-19 Infection in Iranian Children: A Case Series of 9 Patients. Journal of Pediatrics Review. 2020;8(2):139-144.

28. Zheng Y, Huang Z, Ying G, et al. Study of the lymphocyte change between COVID-19 and non-COVID19 pneumonia cases suggesting other factors besides uncontrolled inflammation contributed to multi-organ injury. medRxiv. 2020:2020.2002.2019.20024885. 
29. Zhang G, Hu C, Luo L, et al. Clinical features and outcomes of 221 patients with COVID-19 in Wuhan, China. medRxiv.2020:2020.2003.2002.20030452.

30. Zhao W, Yu S, Zha X, et al. Clinical characteristics and durations of hospitalized patients with COVID19 in Beijing: a retrospective cohort study. medRxiv. 2020:2020.2003.2013.20035436.

31. Bermejo-Martin JF, Almansa R, Menendez R, Mendez R, Kelvin DJ, Torres A. Lymphopenic community acquired pneumonia as signature of severe COVID-19 infection. The Journal of infection.2020;80(5):e23-e24.

32. Tan L, Wang Q, Zhang D, et al. Lymphopenia predicts disease severity of COVID-19: a descriptive and predictive study. Signal Transduct Target Ther. 2020;5(1):33-33.

33. Zheng Y, Huang Z, Ying G, et al. Study of the lymphocyte change between COVID-19 and non-COVID19 pneumonia cases suggesting other factors besides uncontrolled inflammation contributed to multi-organ injury. In: medRxiv; 2020.

34. Ponti G, Maccaferri M, Ruini C, Tomasi A, Ozben T. Biomarkers associated with COVID-19 disease progression. Crit Rev Clin Lab Sci. 2020;57(6):389-399.

35. Zeng F, Huang Y, Guo Y, et al. Association of inflammatory markers with the severity of COVID-19: A meta-analysis. International Journal of Infectious Diseases. 2020;96:467-474.

36. Shenglan P, Xiangyan Z, Daishun L, Baning Y, Jianquan L.Research Square. 2020.

37. Peng L, Liu K-Y, Xue F, Miao Y-F, Tu P-A, Zhou C. Improved Early Recognition of Coronavirus Disease2019 (COVID-19): Single-Center Data from a Shanghai Screening Hospital. Arch Iran Med.2020;23(4):272276.

38. Yuan H CX, Ji X, Du F, Zhou X, He J, et al. A Current emerging respiratory infection: Epidemiological and clinical characteristics, diagnosis and treatments of COVID-19. . Diagnosis and Treatments of COVID19. $(3 / 6 / 2020) 2020$.

39. Yang W, Cao Q, Qin L, et al. Clinical characteristics and imaging manifestations of the 2019 novel coronavirus disease (COVID-19):A multi-center study in Wenzhou city, Zhejiang, China. Journal of Infection. 2020;80(4):388-393.

40. Pan F, Ye T, Sun P, et al. Time Course of Lung Changes at Chest CT during Recovery from Coronavirus Disease 2019 (COVID-19).Radiology. 2020;295(3):715-721.

41. Ng M-Y, Lee EYP, Yang J, et al. Imaging Profile of the COVID-19 Infection: Radiologic Findings and Literature Review. Radiology: Cardiothoracic Imaging. 2020;2(1):e200034.

42. Stojkovic Lalosevic M, Pavlovic Markovic A, Stankovic S, et al. Combined Diagnostic Efficacy of Neutrophil-to-Lymphocyte Ratio (NLR), Platelet-to-Lymphocyte Ratio (PLR), and Mean Platelet Volume (MPV) as Biomarkers of Systemic Inflammation in the Diagnosis of Colorectal Cancer. Dis Markers. 2019;2019:6036979-6036979.

43. Gasparyan AY, Ayvazyan L, Mukanova U, Yessirkepov M, Kitas GD. The Platelet-to-Lymphocyte Ratio as an Inflammatory Marker in Rheumatic Diseases. Ann Lab Med. 2019;39(4):345-357.

44. Qu R, Ling Y, Zhang Y-h-z, et al. Platelet-to-lymphocyte ratio is associated with prognosis in patients with coronavirus disease-19. Journal of Medical Virology. 2020;n/a(n/a).

45. Zhao W, Yu S, Zha X, et al. Clinical characteristics and durations of hospitalized patients with COVID19 in Beijing: a retrospective cohort study. In: medRxiv; 2020.

46. Tahamtan A, Ardebili A. Real-time RT-PCR in COVID-19 detection: issues affecting the results. Expert Rev Mol Diagn.2020;20(5):453-454. 
47. Sethuraman N, Jeremiah SS, Ryo A. Interpreting Diagnostic Tests for SARS-CoV-2. JAMA. 2020;323(22):2249-2251.

Tables :

Table 1: Characteristics and clinical data of the included patients

\begin{tabular}{ll}
\hline Variable & $\mathrm{N}(\%) /$ mean \pm sd \\
\hline Sex; N (\%) & $296(49.7)$ \\
Female & $300(50.3)$ \\
Male & $56.76 \pm 16.47$ \\
Age $($ mean \pm sd) & \\
CT involvement; N (\%) & $30(5)$ \\
$<5$ & $120(20.1)$ \\
$<25$ & $232(38.9)$ \\
$<50$ & $214(35.9)$ \\
$<100$ & $62(10.4)$ \\
CRP; N (\%) & $498(83.6)$ \\
Negative & \\
Positive & $62(10.4)$ \\
ESR; N (\%) & $384(64.4)$ \\
Negative & \\
Positive & $512(85.9)$ \\
Outcome; N (\%) & $84(14.1)$ \\
Discharge & $6.8 \pm 3.5$ \\
Mortality & $213.4 \pm 89.3$ \\
WBC*1000 (mean \pm sd) & $20.35 \pm 10.1$ \\
Platelet $1000($ mean \pm sd $)$ & $1246.7 \pm 709.5$ \\
Lymphocyte in percent $($ mean \pm sd) & $7.79 \pm 4.7$ \\
Lymphocyte in the count $($ mean \pm sd) & \\
Admission time $($ mean \pm sd) &
\end{tabular}

Table 2: Analysis of mortality for patients with COVID-19

\begin{tabular}{llllll}
\hline Lymphopenia & Sever lung involvement $(>50 \%)$ & N $(\%)$ & Mortality & Mortality & OR (CI95\%) \\
\hline & & & NO N $(\%)$ & Yes N (\%) & \\
NO & NO & $46(27.5)$ & $43(93.5)$ & $3(6.5)$ & - \\
NO & YES & $23(13.8)$ & $19(82.6)$ & $4(17.4)$ & $3.49(1.35-8.96)$ \\
YES & NO & $57(34.1)$ & $51(89.5)$ & $6(10.5)$ & $3.62(1.63-8.02)$ \\
YES & YES & $41(24.6)$ & $26(63.4)$ & $15(36.6)$ & $9.24(5.32-19.78)$ \\
\hline
\end{tabular}

Table 3: Analysis of length of stay (LOS) for patients with COVID-19

\begin{tabular}{lllll}
\hline Lymphopenia & Sever lung involvement $(>50 \%)$ & $\mathrm{N}(\%)$ & Mean \pm SD & P-value \\
\hline NO & NO & $210(35.2 \%)$ & $6.7 \pm 4.4$ & 0.000 \\
NO & YES & $74(12.4 \%)$ & $8.2 \pm 4.5$ & \\
YES & NO & $172(28.9 \%)$ & $7.7 \pm 4.3$ & \\
YES & YES & $140(23.5 \%)$ & $9.3 \pm 5.2$ & \\
\hline
\end{tabular}




\section{Figures}

A

B Figure 1: A) A 61-year-old female with COVID-19 pneumonia; Chest CT shows bilateral ground-glass opacities (Blue ar

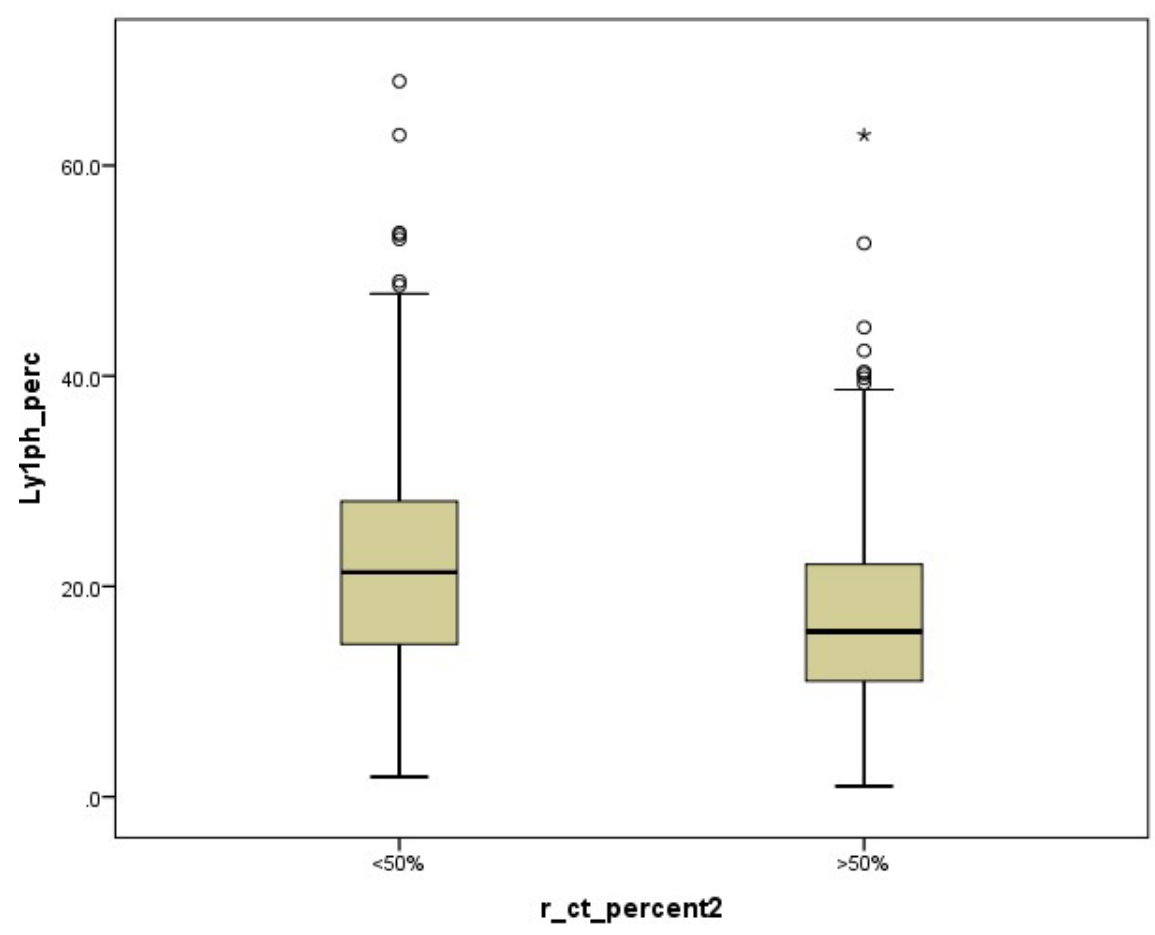

Figure 2: Lymphocyte percentage of COVID-19 patients to the lung involvement 

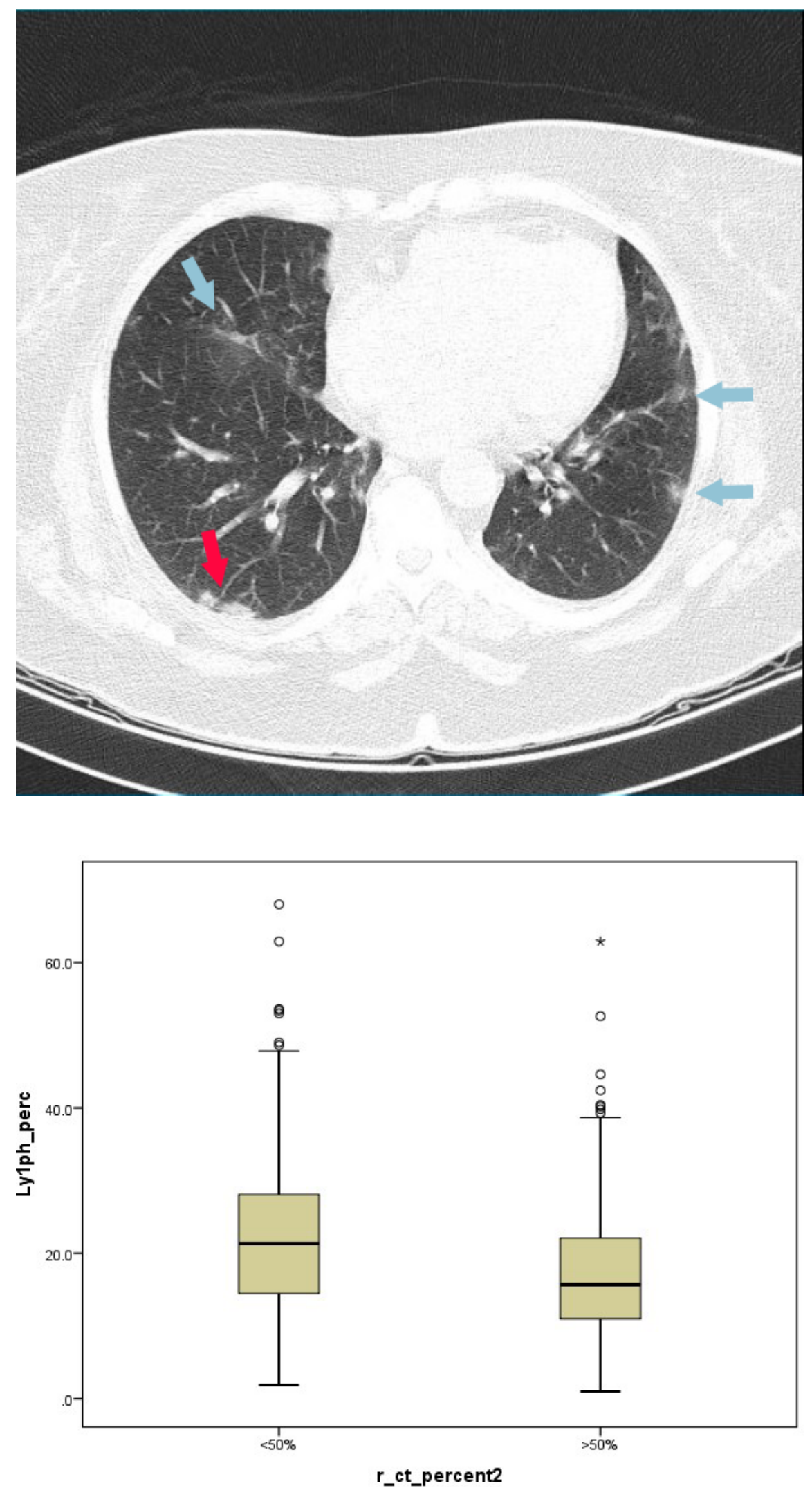


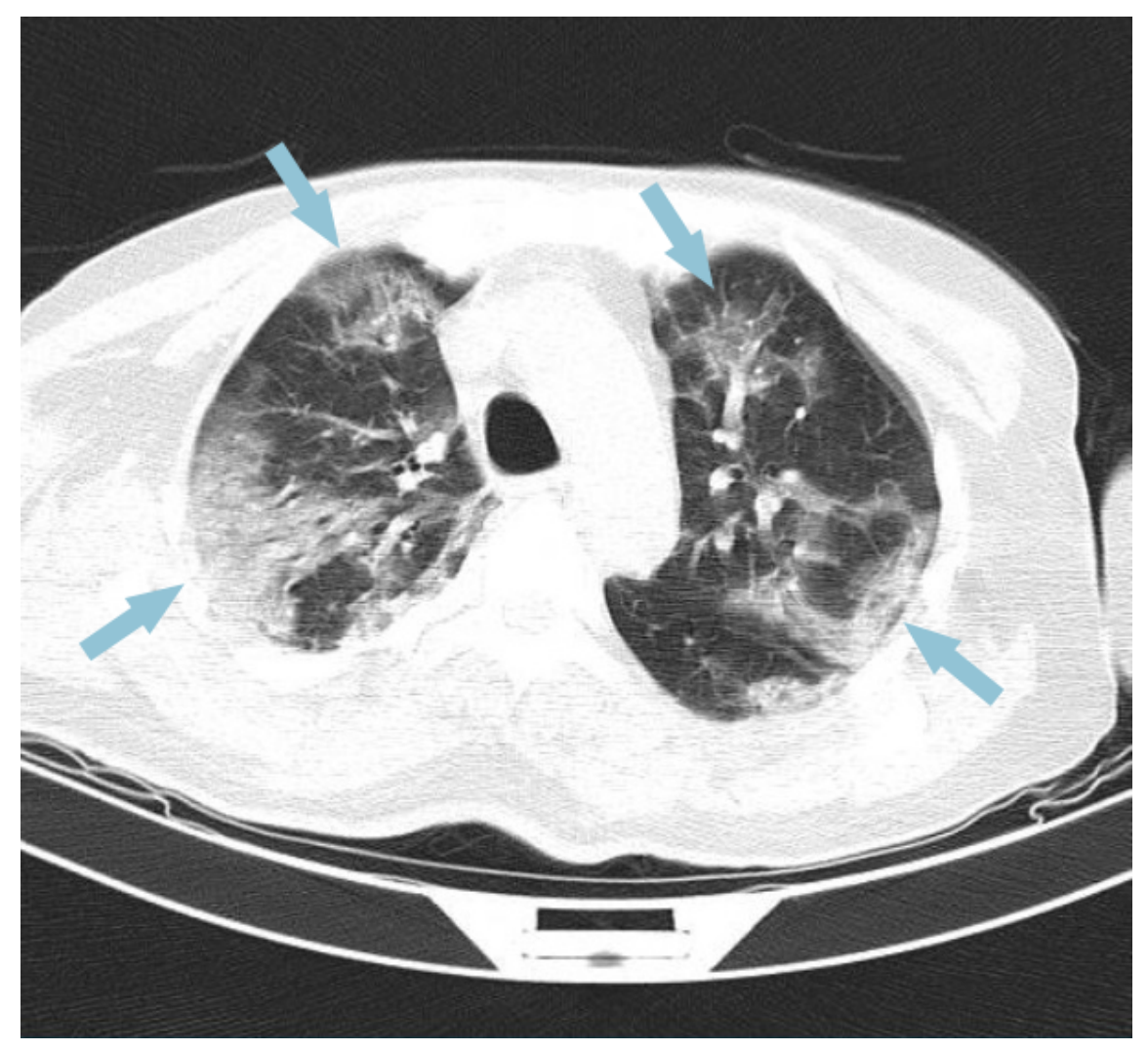

